\title{
Rings close to strongly von Neumann regular
}

\author{
Peter V. Danchev \\ Institute of Mathematics and Informatics, Bulgarian Academy of Sciences,"Acad. G. Bonchev" str., bl. 8, 1113 Sofia, \\ Bulgaria \\ Correspondence to: P.V. Danchev, Email: danchev@math.bas.bg; pvdanchev@yahoo.com
}

\begin{abstract}
We describe the isomorphic structure of the so-called weakly strongly nil-boolean rings that are rings $R$ whose elements $r$ satisfy the relation $r^{2}( \pm 1+q)=r+q$ for some existing nilpotent $q$ which depends on $r$. We prove that the Jacobson radical $J(R)$ in $R$ is a nil-ideal whose elements satisfy special equalities and that either $R / J(R) \cong B$, or $R / J(R) \cong \mathbb{Z}_{3}$, or $R / J(R) \cong B \times \mathbb{Z}_{3}$, where $B$ is a Boolean ring. This somewhat supplies our recent investigations in Irish Math. Soc. Bull. (2015), Tsukuba J. Math. (2016), Rend. Sem. Mat. Univ. Politech. Torino (2018) and Internat. Balkan J. Math., retitled as Eurasian Bull. Math. (2018).
\end{abstract}

Keywords: Boolean rings, Strongly regular rings, Finite fields, Jacobson radical.

\section{Introduction}

Everywhere in the text of the current paper, all our rings $R$ are assumed to be associative, containing the identity element 1 , which differs from the zero element 0 of $R$. Our standard terminology and notations are mainly in agreement with [8]. For instance, to be more exact, $U(R)$ denotes the set of all units in $R$, $\operatorname{Nil}(R)$ the set of all nilpotents in $R$ and $J(R)$ the Jacobson radical of $R$.

The well-known notion of Boolean rings states that each element in these rings is an idempotent. These rings are successfully classified as the subdirect product of the field $\mathbb{Z}_{2}$. Several other generalizations of this subject were presented in [1, 2]-6] and 7], respectively. Specifically, in [1] and in [7] plus [4 were independently characterized those rings $R$, calling them weakly boolean, whose elements are solutions of the equations $x^{2}=x$ or $x^{2}=-x$. It was established there that either $R \cong B$, or $R \cong \mathbb{Z}_{3}$, or $R \cong B \times \mathbb{Z}_{3}$. Moreover, in [2] and, respectively in [3], were explored rings of the type $r(1+q) r=r$ and $r^{2}(1+q)=r$ which are showed to be Boolean rings and, respectively, rings of the type $r( \pm 1+q) r=r$ and $r^{2}( \pm 1+q)=r$ which are showed to be the aforementioned weakly boolean rings. These rings are hopefully closely related to the well-known (strongly) regular rings $R$ (see, e.g., 9]) satisfying for all their elements $r \in R$ the equalities $r a r=r$ (resp., $\left.r^{2} a=r\right)$ for some existing element $a$ in $R$ which depends on $r$.

It is, therefore, rather natural to continue the consideration of rings of some special sorts of the above kind. So, we come to our new key point of view, which is a common generalization of the classical concept of Boolean rings.

Definition 1.1. We shall say that the ring $R$ is strongly nil-boolean if, for each $r \in R$, there exists $q \in \operatorname{Nil}(R)$ with $r^{2}(1+q)=r+q$ and that $R$ is weakly strongly nil-boolean provided $r^{2}( \pm 1+q)=r+q$.

The purpose motivated the writing up of this short article is to describe up to an isomorphism the stated above two classes of rings, and thus to support the aforementioned results studied in this branch. This will be done in the subsequent section.

\section{The Main Result}

We will prove now the following assertion.

Theorem 2.1. Let $R$ be a weakly strongly nil-boolean ring. Then $J(R)$ is nil and either $R / J(R) \cong B$ is Boolean, or $R / J(R) \cong \mathbb{Z}_{3}$, or $R / J(R) \cong B \times \mathbb{Z}_{3}$.

In particular, if $R$ is strongly nil-boolean, then $J(R)$ is nil and $R / J(R) \cong B$ is Boolean.

Proof. For the element $r=2$ substituted in the equality from Definition 1.1 , we have that $2^{2}( \pm 1+q)=2+q$ for some nilpotent $q$, whence $4(1+q)=2+q$ or $4(-1+q)=2+q$. Thus $2=-3 q \in N i l(R)$ or 
$6=3 q \in \operatorname{Nil}(R)$. Finally, in both cases, $6 \in \operatorname{Nil}(R)$. Consequently, the Chinese Remainder Theorem yields the decomposition $R \cong R_{1} \times R_{2}$ for some rings $R_{1}$ and $R_{2}$, where $R_{1}=\{0\}$ or $2 \in \operatorname{Nil}\left(R_{1}\right)$ as well as $R_{2}=\{0\}$ or $3 \in \operatorname{Nil}\left(R_{2}\right)$. Routine coordinate-wise arguments show that both $R_{1}, R_{2}$ are also weakly strongly nil-boolean rings.

We foremost shall consider the partial cases when $2=0$ in $R_{1}$ and when $3=0$ in $R_{2}$; so $6=0$ in $R$.

Case 1: "Consider $R_{1}$ ": For any $r_{1} \in R_{1}$ it must be that $r_{1}^{2}\left(1+q_{1}\right)=r_{1}+q_{1}$, for some $q_{1} \in \operatorname{Nil}\left(R_{1}\right)$. Consequently, replacing $r_{1}$ by $r_{1}+1$ in this equation, we arrive at $\left(r_{1}+1\right)^{2}\left(1+t_{1}\right)=\left(r_{1}+1\right)+t_{1}$ for some existing $t_{1} \in \operatorname{Nil}\left(R_{1}\right)$, i.e., we deduce that $\left(r_{1}^{2}+1\right)\left(1+t_{1}\right)=\left(r_{1}+1\right)+t_{1}=r_{1}+\left(1+t_{1}\right)$ because $2=0$. Hence $r_{1}^{2}\left(1+t_{1}\right)=r_{1}$. We claim that $N i l\left(R_{1}\right)=\{0\}$. To that aim, given $d_{1} \in R_{1}$ with $d_{1}^{2}=0$, it follows that $d_{1}^{2}\left(1+t_{1}\right)=d_{1}$, that is exactly, $d_{1}=0$, as pursued. That is why, $r_{1}^{2}=r_{1}$ which means that $R_{1}$ is Boolean, as stated.

Case 2: "Consider $R_{2}$ ": For any $r_{2} \in R_{2}$ it has to be that $r_{2}^{2}\left(1+q_{2}\right)=r_{2}+q_{2}$ or that $r_{2}^{2}\left(-1+q_{2}\right)=r_{2}+q_{2}$, for some $q_{2} \in \operatorname{Nil}\left(R_{2}\right)$. It is, therefore, obvious that if $v_{2} \in R_{2}$ with $v_{2}^{2}=1$, then either $v_{2}=1$ or $v_{2}=-1$. So, for each idempotent $e_{2} \in R_{2}$, we derive that $2 e_{2}-1=1$ or that $2 e_{2}-1=-1$. Thus either $e_{2}=1$ or $e_{2}=0$ as $3=0$ and $2 e_{2}=-e_{2}$. This allows us to infer that $R_{2}$ is strongly indecomposable in the sense that it does not possess non-trivial idempotents.

Moreover, $\left(r_{2}+1\right)^{2}\left(1+t_{2}\right)=\left(r_{2}+1\right)+t_{2}$ or $\left(r_{2}+1\right)^{2}\left(-1+t_{2}\right)=\left(r_{2}+1\right)+t_{2}$ for some existing $t_{2} \in \operatorname{Nil}\left(R_{2}\right)$. The first equality implies that $r_{2}^{2}\left(1+t_{2}\right)=r_{2}\left(t_{2}-1\right)$, and the second one that $r_{2}^{2}\left(-1+t_{2}\right)=r_{2} t_{2}-1$. We now assert that $\operatorname{Nil}\left(R_{2}\right)=\{0\}$. To illustrate this, given $d_{2} \in R_{2}$ with $d_{2}^{2}=0$, it follows that $d_{2}\left(t_{2}-1\right)=0$ or that $d_{2} t_{2}=1$. Therefore, in both situations, by taking into account that $t_{2}-1 \in U\left(R_{2}\right)$, it readily follows that $d_{2}=0$, as required, as the second equality $d_{2} t_{2}=1$ is totally impossible forcing that $0=1$. Furthermore, $r_{2}^{2}=-r_{2}$ or $r_{2}^{2}=1$ yielding actually with the aid of [7] (or by virtue of [1]) that $R_{2} \cong \mathbb{Z}_{3}$, as asked for (and thus we also receive $r_{2}^{2}= \pm r_{2}$ for all $r_{2} \in R_{2}$ by replacing $r_{2} \rightarrow r_{2}-1$ and bearing in mind that $3=0)$. Nevertheless, another useful argument, in order to conclude that $R_{2}$ is the three element field, is like this: $r_{2}^{2}=-r_{2}$ along with $r_{2}^{2}=1$ (same as $r_{2}^{2}= \pm r_{2}$ ) enable us that $r_{2}^{3}=r_{2}$ and, therefore, we exploit 8 to extract that $R_{2}$ is a subdirect product of family of copies of rings isomorphic to $\mathbb{Z}_{3}$. However, by what we have just shown above, the ring $R_{2}$ does not have non-trivial idempotents. This allows us to conclude that $R_{2} \cong \mathbb{Z}_{3}$ after all.

We are now ready to handle the general case. To do that, it is elementarily to see that $R \cong R_{1} \times R_{2}$ will imply that $J(R) \cong J\left(R_{1}\right) \times J\left(R_{2}\right)$ and also that $R / J(R) \cong\left[R_{1} / J\left(R_{1}\right)\right] \times\left[R_{2} / J\left(R_{2}\right)\right]$, where the first direct factor is of characteristic 2 , and the latter one is of characteristic 3 , because $2 \in J\left(R_{1}\right)$ and $3 \in J\left(R_{2}\right)$. Henceforth, we may employ Cases 1 and 2 alluded to above to get the desired isomorphism result. The second stated particular isomorphism description is now an immediate consequence of what we have already proved so far.

As to show next that $J(R)$ is nil, given an arbitrary element $z \in J(R)$ such that $z^{2}( \pm 1+q)=z+q$ for some existing $q \in \operatorname{Nil}(R)$. We differ two cases, namely $z^{2}(1+q)=z+q$ or $z^{2}(-1+q)=z+q$. In the first possibility, we write $z^{2}-z=\left(1-z^{2}\right) q$, i.e., $-(1-z) z=(1-z)(1+z) q$. Since $1-z \in U(R)$, it follows by canceling on the left that $-z=q+z q$ and hence that $z(q+1)=-q$. Finally, $z=-q(q+1)^{-1} \in \operatorname{Nil}(R)$, as expected.

In the second situation, we write $z^{2}+z=\left(z^{2}-1\right) q$, that is, $(z+1) z=(z+1)(z-1) q$. Since $z+1 \in$ $U(R)$, we may cancel the identity again on the left, so that $z=z q-q$ whence $z(q-1)=q$. Finally, $z=q(q-1)^{-1} \in N i l(R)$, as promised. This proves our claim about the nil property of $J(R)$.

It is worthwhile to add the following comments:

Remark 2.2. In general, according to the results from [7, weakly strongly nil-boolean rings are themselves weakly nil clean, that is, each element is a sum or a difference of a nilpotent and an idempotent. Indeed, invoking Theorem 2.1 for any $r \in R$ it is fulfilled that $r^{2}-r \in J(R)$ or $r^{2}+r \in J(R)$. Since $J(R)$ is nil, there is an idempotent $e \in R$ such that $e-r \in J(R) \subseteq N i l(R)$ or $e+r \in J(R) \subseteq N i l(R)$ which ensure that $r \in I d(R)+N i l(R)$ or $r \in-I d(R)+N i l(R)$, as required, substantiating our claim.

However, if $2=0$ in strongly nil-boolean rings, they are necessarily Boolean; while if $6=0$ in weakly strongly 
nil-boolean rings, they are of necessity weakly boolean. In fact, one sees that $J(R)=\{0\}$ whenever $6=0$ and we just need to apply Theorem 2.1.

We end our work with a series of problems as follows:

Problem 2.3. Describe (weakly) nil-boolean rings that are rings $R$ for which, for every $r \in R$, the relation $r(1+q) r=r+q$ (resp., $r( \pm 1+q) r=r+q$ ) holds, for some $q \in N i l(R)$ depending on $r$.

The next query is a slight restatement from [2]. Consulting with [7 for more account, let us recall that a ring is said to be nil clean if all its elements are sums of a nilpotent and an idempotent, whereas it is said to be weakly nil clean if all its elements are sums or differences of a nilpotent and an idempotent.

Problem 2.4. Characterize all rings $R$ such that, for every $r \in R$, there exist a nilpotent $q \in R$ and an idempotent $e \in R$ with the property $r(e+q) r=r$ or, more generally, even that $r( \pm e+q) r=r$. Are these rings nil clean or, respectively, weakly nil clean?

In closing, as a simple union of the previous two questions, we can state the following.

Problem 2.5. In the presence of above notations, classify all rings $R$ whose elements satisfy the equalities $r(e+q) r=r+q$ or $r( \pm e+q) r=r+q$, respectively. What is their relationship with the classes of (weakly) nil clean rings?

\section{Methods}

We mainly have used a straightforward machinery to obtain the chief result described above.

\section{Results}

The basic result established above states that (weakly) strongly nil-boolean rings are characterizable in terms of Boolean rings, the field $\mathbb{Z}_{3}$ eventually, and nil Jacobson radicals only.

\section{Discussion}

Our results stated above are somewhat a natural continuation of previous author's achievements listed below in the bibliography.

\section{Acknowledgement}

The author owes his sincere thanks to the reviewer for the careful refereeing of the paper.

\section{References}

[1] Ahn, M.-S. and Anderson, D.D., 2006, Weakly clean rings and almost clean rings, Rocky Mountain J. Math., 36, 783-798.

[2] Danchev, P.V., 2015, A new characterization of boolean rings with identity, Irish Math. Soc. Bull., 76, $55-60$.

[3] Danchev, P.V., 2016, Weakly UU rings, Tsukuba J. Math., 40, 101-118.

[4] Danchev, P.V., 2017, Weakly semi-boolean unital rings, JP J. Algebra, Numb. Th. \& Appl., 39, 261-276.

[5] Danchev, P.V., 2018, Quadratic equations and extensions of boolean rings, Rend. Sem. Mat. Univ. Politech. Torino, 76, 33-39.

[6] Danchev, P.V., 2018, Rings whose elements satisfy quadratic equations, Internat. Balkan J. Math. (= Eurasian Bull. Math.), 1, 62-68. 
[7] Danchev, P.V. and McGovern, W.Wm., 2015, Commutative weakly nil clean unital rings, J. Algebra, $425,410-422$.

[8] Lam, T.Y., 2001, A First Course in Noncommutative Rings, Second Edition, Graduate Texts in Math., vol. 131, Springer-Verlag, Berlin-Heidelberg-New York.

[9] Tuganbaev, A., 2002, Rings Close to Regular, Mathematics and Its Applications, vol. 545, Kluwer Academic Publishers, Dordrecht. 